\title{
O uso de ferramentas computacionais na construção de contraexemplos para um teorema de Fiedler-Nikiforov sobre grafos hamiltonianos
}

Anderson Luiz P. Porto (D)

\author{
Marcelle Fernanda R. Xavier (D)
}

Douglas F. G. Santiago

\begin{abstract}
Resumo
Neste artigo são exibidos contraexemplos para a recíproca de um resultado de Fiedler-Nikiforov que trata de uma desigualdade envolvendo os conceitos de raio espectral de um grafo e sua hamiltonicidade. Para tanto, dois métodos distintos foram utilizados para obter os resultados. O primeiro foi feito baseado em resultados dados pelo Scilab que determinou a não validade da inequação de Fiedler-Nikiforov. No segundo, foram usados conceitos e resultados básicos de análise matemática. Além disso, utilizou-se dos conceitos de clique máximo de um grafo e sua relação com o raio espectral para a construção dos contraexemplos. Este trabalho foi realizado no período compreendido entre os anos de 2017 e 2019 e fez parte de um projeto Picme em que a segunda autora desenvolveu sua pesquisa e monografia na área de Teoria de Grafos.
\end{abstract}

Palavras-chave: Grafos; Matriz de adjacência; Hamiltonianos; Raio espectral; Teorema de Bolzano.

\section{Abstract}

In this article, counterexamples are presented for the reciprocal of a Fiedler-Nikiforov result that deals with an inequality involving the concepts of spectral radius of graphs and its hamiltonicity. For this, two different methods were used to obtain the results. The first was made based on results given by Scilab that determined the non-validity of the Fiedler-Nikiforov inequality. In the second, basic concepts and results of mathematical analysis were used. In addition, the concepts of maximum click of a graph and its relation to the spectral radius were used to construct counterexamples. This work was carried out in the period between the years 2017 and 2019 and was part of a PICME project in which the second author developed her research and monograph in the area of Graph Theory.

Keywords: Graphs; Adjacency matrix; Hamiltonians; Spectral radius; Bolzano's theorem.

\section{Introdução}

A teoria dos grafos teve início em 1736 quando Leonard Euler resolveu o famoso problema das sete pontes, que intrigava a população da antiga cidade de Königsberg na Prússia, atualmente Kaliningrado na Rússia. Euler representou o problema com uma estrutura geométrica que hoje conhecemos como o grafo de Euler das sete pontes. O rio Pregel passava pelo interior da cidade e 
a dividia em quatro partes, formando um complexo de ilhas interligadas por sete pontes, conforme a Figura 1 [2]. Uma representação do problema na forma de um grafo encontra-se na Figura 2.

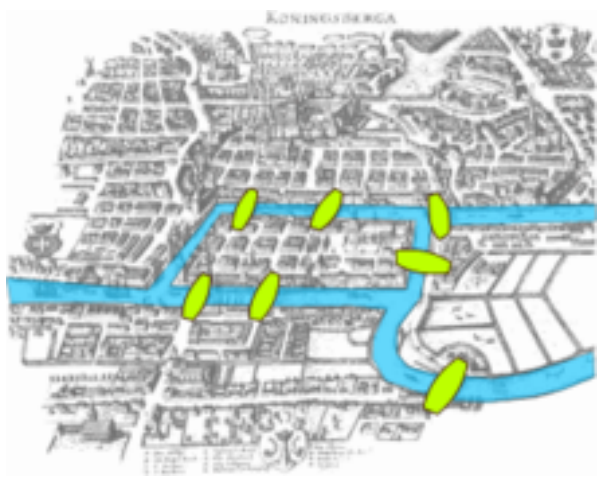

Figura 1: Königsberg Bridges.

Fonte: https://en.wikipedia.org/wiki/File:Konigsberg_bridges.png.

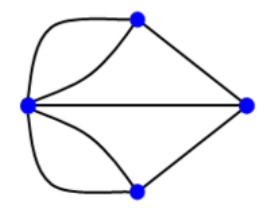

Figura 2: Grafo das ilhas de Königsberg.

Fonte: https://pt.wikipedia.org/wiki/Ficheiro:K\%C3\%B6nigsberg_graph.png.

O estudo sobre grafos hamiltonianos teve início em 1858, quando o matemático irlandês sir William Rowan Hamilton (1805 - 1865), criou um jogo matemático envolvendo um dodecaedro regular, onde se pretendia encontrar um ciclo que passasse por todos os vértices sem repeti-los. Tal problema também pode ser visualizado a partir de um grafo. Grafos que contêm tais ciclos são ditos grafos hamiltonianos ou grafos de Hamilton (Adaptado de [2]).

Um laço é um grafo com uma única aresta que se conecta a um único vértice (ver Definição 3). Um grafo completo com n vértices é um grafo sem laços tal que cada um de seus vértices está conectado a todos os outros n-1 vértices não adjacentes por uma única aresta. Tais grafos serão denotados por $\mathrm{K}_{\mathrm{n}}$. Por exemplo, um triângulo (denotado por $\mathrm{K}_{3}$ ) é um grafo completo com 3 vértices, enquanto que um quadrado com suas diagonais é um grafo completo com 4 vértices, denotado por $\mathrm{K}_{4}$.

Dois tipos de grafos serão as exceções no teorema a seguir. O primeiro é construído a partir da união de um grafo completo contendo $n-1$ vértices e um vértice isolado $v$ (isto é, um vértice que não está contido no grafo completo). O segundo é obtido a partir da união de um grafo completo contendo $\mathrm{n}-1$ vértices e uma aresta e que não está contida no conjunto de arestas do grafo completo. O primeiro grafo será denotado por $\mathrm{K}_{\mathrm{n}-1} \cup \mathrm{v}$ e o segundo por $\mathrm{K}_{\mathrm{n}-1}$ 巴 e, respectivamente. Os símbolos distintos para a adição de vértices e arestas foram feitos para distinguir os dois tipos 
de construções: a primeira, no conjunto de vértices, e a segunda, no conjunto de arestas assim como no conjunto de vértices. Veja a Figura 3 para representações na forma de grafos:

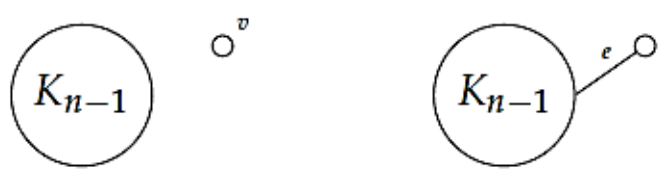

Figura 3: Grafos $\mathrm{K}_{\mathrm{n}-1} \cup \mathrm{v}$ e $\mathrm{K}_{\mathrm{n}-1}$ 巴 e da esquerda para a direita. Fonte: Santos [11].

A matriz de adjacência de um grafo $G$, denotada por $A(G)=\left(a_{i j}\right)$, é uma matriz quadrada cujas entradas satisfazem $a_{i j}=1$, se o i-ésimo vértice de $G$ é conectado por uma aresta ao j-ésimo vértice, e $\mathrm{a}_{\mathrm{ij}}=0$, nos casos contrários. O raio espectral de um grafo $\mathrm{G}$, denotado por $\mu(\mathrm{G})$, é o maior autovalor positivo de $\mathrm{A}(\mathrm{G})$.

No artigo Spectral radius and hamiltonicity of graphs, os autores Fiedler e Nikiforov provaram o seguinte:

Teorema 1 ([4]). Seja $\mathrm{G}$ um grafo com $\mathrm{n}$ vértices e raio espectral $\mu(\mathrm{G})$. Se $\mu(\mathrm{G}) \geq \mathrm{n}-2$, então $\mathrm{G}$ contém um caminho hamiltoniano, exceto se $\mathrm{G}$ for igual a $\mathrm{G}=\mathrm{K}_{\mathrm{n}-1} \cup \mathrm{v}$. Se a inequação é estrita, então $\mathrm{G}$ contém um ciclo hamiltoniano, exceto se $\mathrm{G}=\mathrm{K}_{\mathrm{n}-1}$ ש e.

Este artigo possui dois propósitos principais. O primeiro é apresentar contraexemplos para a recíproca do resultado acima, que nem sempre são bem explorados, justificando-os de duas maneiras distintas: a primeira através do cálculo direto do raio espectral usando o software Scilab ([12]) e a segunda mediante resultados analíticos com o auxílio de gráficos construídos pelo software GeoGebra ([5]). A construção dos contraexemplos foi baseada em uma desigualdade que envolve o conceito de clique máximo de um grafo, que será discutido na seção 3 deste artigo [2].

O segundo propósito é evidenciar aspectos e ferramentas da investigação matemática que podem e devem ser utilizados como facilitadores no desenvolvimento dos argumentos aplicados na construção dos contraexemplos, particularmente os recursos computacionais.

Esses mecanismos auxiliam tanto o ensino quanto o desenvolvimento da matemática propriamente dita, sendo comum o uso de softwares como Geogebra ([5]), Scilab ([12]), Octave, Matlab, Maple, yEd, Graphviz ([6]), entre outros. Tais ferramentas permitem a observação de exemplos, possibilitando propor conjecturas ou mesmo refutá-las e estabelecer a não validade de resultados, como é feito neste artigo com a recíproca do Teorema 1. As ferramentas computacionais atualmente representam ótimos recursos investigativos dentro da matemática e em diversas outras áreas do conhecimento. Seus resultados podem até mesmo atuar como provas válidas ou sugerir caminhos para que essas provas sejam construídas, necessitando portanto de uma complementação teórica mais formal nesses casos. Neste texto, os recursos computacionais serão utilizados para: i) encontrar autovalores de polinômios característicos de grafos, usando-se o Scilab; ii) analisar graficamente o comportamento dos polinômios característicos dos grafos através do GeoGebra e iii) desenhar alguns grafos a partir dos softwares yEd ([16]) e Graphviz ([6]).

A segunda autora foi medalhista da Obmep de 2011 a 2013 e participou do programa PICJr da Obmep, neste período, no polo de Diamantina-MG sob a supervisão do primeiro autor. Ao ingres- 
sar na UFVJM a aluna desenvolveu sua pesquisa na área de Álgebra Linear e Teoria Clássica e Espectral de Grafos, estudos relacionados ao programa Picme com o mesmo professor orientador. O terceiro autor participou de forma colaborativa em todas as etapas. Os autores iniciaram, a partir de 2018, um estudo sobre vários softwares de matemática cujo objetivo principal foi encontrar contraexemplos para o Teorema de Fiedler-Nikiforov. Por fim, escreveu-se uma monografia intitulada: Um Teorema de Fiedler-Nikiforov sobre a Hamiltonicidade de Grafos Simples e Conexos $(\operatorname{ver}[18])$.

\section{Definições, exemplos e fatos elementares}

Será feita uma breve revisão dos conceitos e fatos elementares da teoria clássica de grafos, bem como da teoria espectral de grafos. O leitor que tiver interessado em detalhes deve consultar os livros [2] e [7] para a teoria clássica de grafos. Para a teoria espectral, consulte [1] e [3] como exemplos.

Definição 1 (Grafo não orientado). Um grafo não orientado $G=(V(G), E(G), \Psi(G))$ é um trio de objetos composto por um conjunto não vazio de vértices, denotado por $\mathrm{V}(\mathrm{G})$; um conjunto de arestas, denotado por $\mathrm{E}(\mathrm{G})$, esse podendo ser vazio; e uma relação entre tais conjuntos, que é dada pela função de incidência $\Psi_{\mathrm{G}}: \mathrm{E}(\mathrm{G}) \rightarrow \mathrm{V}(\mathrm{G}) \otimes \mathrm{V}(\mathrm{G})$ que aplica cada aresta $\mathrm{f} \in \mathrm{E}(\mathrm{G}) \mathrm{em}$ $\Psi_{\mathrm{G}}(\mathrm{f})=[\mathrm{u}, \mathrm{v}]=\mathrm{uv}=[\mathrm{v}, \mathrm{u}]$, onde $\mathrm{u}$ e $\mathrm{v}$ são chamados de vértices adjacentes ou incidentes na aresta f, e o símbolo $\otimes$ denota o conjunto dos pares não ordenados, isto é, o conjunto dos pares que satisfazem a relação $[\mathrm{u}, \mathrm{v}]=[\mathrm{v}, \mathrm{u}]$.

Um grafo não orientado pode apresentar diversas realizações geométricas. No entanto, todas elas implicam grafos isomorfos. Portanto vamos nos referir ao grafo como sendo uma de suas realizações geométricas, ficando implícito, como na Figura 4, os 3 objetos mencionados na definição.

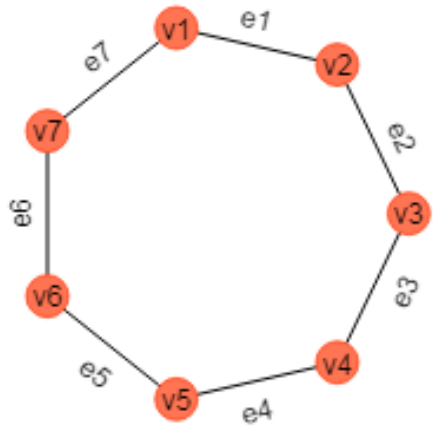

Figura 4: Grafo G e suas incidências.

Observem que no grafo da Figura 4 temos:

- $\Psi_{\mathrm{G}}\left(\mathrm{e}_{1}\right)=\left[\mathrm{v}_{1}, \mathrm{v}_{2}\right]=\left[\mathrm{v}_{2}, \mathrm{v}_{1}\right]$, assim $\mathrm{v}_{1}$ e $\mathrm{v}_{2}$ são incidências de $\mathrm{e}_{1}$; além disso, $\mathrm{v}_{1}$ e $\mathrm{v}_{2}$ são vértices adjacentes.

- $\Psi_{\mathrm{G}}\left(\mathrm{e}_{2}\right)=\left[\mathrm{v}_{2}, \mathrm{v}_{3}\right]$, logo $\mathrm{v}_{2}$ e $\mathrm{v}_{3}$ são incidências de $\mathrm{e}_{2} ;$ almdisso, $\mathrm{v}_{2}$ e $\mathrm{v}_{3}$ são vértices adjacentes. Note que $e_{1}$ e $e_{2}$ são arestas adjacentes, pois incidem em $v_{2}$. 
Definição 2 (Grafo finito). Um grafo é dito finito se ambos os conjuntos $V(G)$ e $E(G)$ são finitos. Em geral, neste texto trataremos somente de grafos finitos.

Definição 3 (Laço). Se uma aresta tem as mesmas incidências, isto é, $\Psi_{G}\left(e_{1}\right)=\left[v_{1}, v_{1}\right]$ para algum vértice $\mathrm{v}_{1}$, então está aresta será chamada de um laço do grafo.

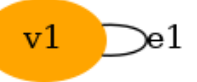

Figura 5: Laço.

Definição 4 (Arestas paralelas). Quando duas ou mais arestas possuem as mesmas extremidades, ou seja, satisfazem: $\Psi_{G}\left(e_{1}\right)=\Psi_{G}\left(e_{2}\right)=v_{1} v_{2}$, e $e_{1} \neq e_{2}$, tais arestas serão chamadas arestas paralelas.

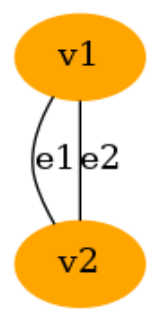

Figura 6: Arestas paralelas..

Definição 5 (Grafo simples). Se um grafo não possui laços ou arestas paralelas ele é chamado de grafo simples.

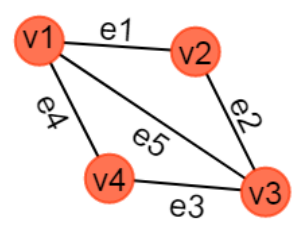

(a) Grafo simples.

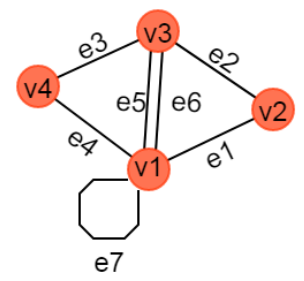

(b) Grafo não simples.

Figura 7: Grafo simples e não simples.

Definição 6 (Subgrafo). Seja $\mathrm{G}_{1}=\left(\mathrm{V}_{1}, \mathrm{E}_{1}\right)$ e $\mathrm{G}_{2}=\left(\mathrm{V}_{2}, \mathrm{E}_{2}\right)$ dois grafos. Diz-se que $\mathrm{G}_{2}$ é um subgrafo de $G_{1}$ se $V_{2} \subseteq V_{1}, E_{2} \subseteq E_{1}$ e a função de incidência de $G_{2}$ é igual à função de incidência de $G_{1}$ restrita ao conjunto de arestas $E_{2}$. Pode-se dizer também que $G_{1}$ é um supergrafo de $G_{2}$. 
Definição 7 (Caminho). Define-se um caminho em um grafo $G$ como sendo uma sequência finita que alterna vértices distintos $\left(\mathrm{v}_{\mathrm{j}}\right)$ e arestas distintas $\left(\mathrm{e}_{\mathrm{k}}\right)$ do grafo $\mathrm{G}$, que pode ser dos tipos $\mathrm{W}=\mathrm{v}_{0}$ (caminho trivial) ou $\mathrm{W}=\mathrm{v}_{0} \mathrm{e}_{1} \mathrm{v}_{1} \mathrm{e}_{2} \cdots \mathrm{v}_{\mathrm{n}-1} \mathrm{e}_{\mathrm{n}} \mathrm{v}_{\mathrm{n}}$ (caminho de comprimento $\mathrm{n}$ ).

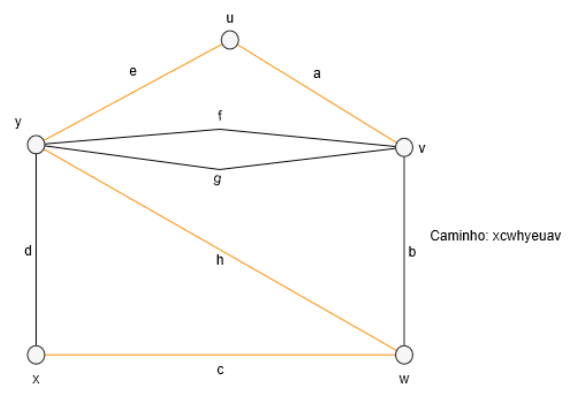

Figura 8: Exemplo de um caminho (path).

Fonte: Adaptado de Bond-Murty, p. 12, 1982.

Definição 8 (Ciclos). Define-se um ciclo em um grafo $G$ como sendo uma sequência finita que alterna vértices distintos $\left(\mathrm{v}_{\mathbf{j}}\right)$ e arestas distintas $\left(\mathrm{e}_{\mathrm{k}}\right)$ do grafo $\mathrm{G}$, a menos dos vértices inicial e final, dos tipos $\mathrm{W}=\mathrm{v}_{0}$ (ciclo trivial) ou $\mathrm{W}=\mathrm{v}_{0} \mathrm{e}_{1} \mathrm{v}_{1} \mathrm{e}_{2} \cdots \mathrm{v}_{\mathrm{n}-1} \mathrm{e}_{\mathrm{n}} \mathrm{v}_{0}$ (ciclo de comprimento $\mathrm{n}$ ou um n-ciclo). Um grafo sem ciclos é chamado de acíclico.

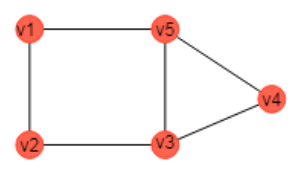

Figura 9: Exemplos de ciclos.

Na Figura 9, a sequência $T_{1}=v_{1} v_{2} v_{3} v_{4} v_{5} v_{3} v_{2} v_{1}$ não é um ciclo, enquanto que as demais sequências a seguir são ciclos: $\mathrm{C}_{1}=\mathrm{v}_{1} \mathrm{v}_{5} \mathrm{v}_{4} \mathrm{v}_{3} \mathrm{v}_{2} \mathrm{v}_{1}$ (um 5-ciclo), $\mathrm{C}_{2}=\mathrm{v}_{4} \mathrm{v}_{3} \mathrm{v}_{5} \mathrm{v}_{4}$ (um 3-ciclo ou triângulo) e $\mathrm{C}_{3}=\mathrm{v}_{1} \mathrm{v}_{5} \mathrm{v}_{3} \mathrm{v}_{2} \mathrm{v}_{1}$ (um 4-ciclo).

Definição 9 (Grafo completo). É um grafo simples que possui todos os seus pares de vértices distintos conectados por uma única aresta. Denotaremos por $\mathrm{K}_{\mathrm{n}}$ o grafo completo com $\mathrm{n}$ vértices. 

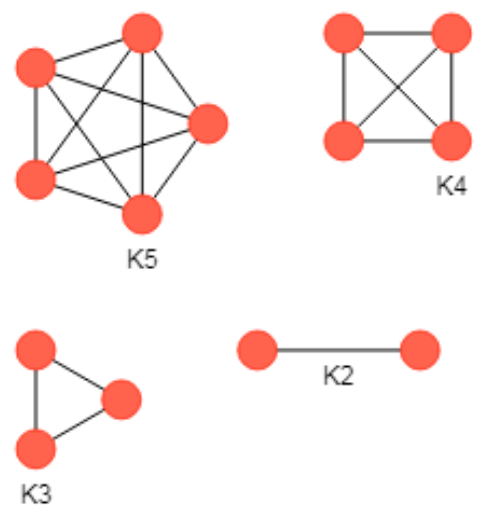

K1

Figura 10: Grafos completos.

Definição 10 (Matriz de adjacência de um grafo). Seja G um grafo com n vértices numerados de 1 a n. Definimos a matriz de adjacência de $G$, com respeito a essa ordenação de seus vértices, como sendo a matriz quadrada $n \times n$, onde cada entrada da matriz satisfaz $a_{i j}=1$ se $v_{i}$ e $v_{j}$ são adjacentes, e $a_{i j}=0$, caso contrário. Notação: $A(G)$ ou simplesmente A quando não houver confusão com respeito ao grafo e à sua ordenação.

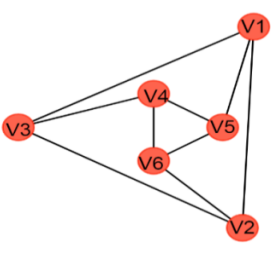

Figura 11: Grafo G.

A matriz de adjacência do grafo $\mathrm{G}$ é a seguinte:

$$
\mathrm{A}(\mathrm{G})=\left(\begin{array}{cccccc}
0 & 1 & 1 & 0 & 1 & 0 \\
1 & 0 & 1 & 0 & 0 & 1 \\
1 & 1 & 0 & 1 & 0 & 0 \\
0 & 0 & 1 & 0 & 1 & 1 \\
1 & 0 & 0 & 1 & 0 & 1 \\
0 & 1 & 0 & 1 & 1 & 0
\end{array}\right)
$$

Note que a matriz de adjacência de um grafo é uma matriz real simétrica e independe da ordenação dos seus vértices [3]. Portanto tais matrizes possuem todos os seus autovalores reais [8]. O polinômio característico de uma matriz de adjacência de um grafo apresenta algumas propriedades interessantes:

Proposição $1([1])$. Seja $\mathrm{G}$ um grafo com $\mathrm{n}$ vértices e $\mathrm{m}$ arestas e considere $\mathrm{p}_{\mathrm{G}}(\mathrm{x})=\mathrm{x}_{\mathrm{n}}+\mathrm{a}_{1} \mathrm{x}^{\mathrm{n}-1}+$ 
$\mathrm{a}_{2} \mathrm{x}^{\mathrm{n}-2}+\mathrm{a}_{3} \mathrm{x}^{\mathrm{n}-3}+\ldots+\mathrm{a}_{\mathrm{n}-1} \mathrm{x}+\mathrm{a}_{\mathrm{n}}$ o polinômio característico de $\mathrm{G}$. Então os coeficientes de $\mathrm{p}_{\mathrm{G}}(\mathrm{x})$ satisfazem: i) $\mathrm{a}_{1}=0$; ii) $\mathrm{a}_{2}=-\mathrm{m}$; iii) $\mathrm{a}_{3}=-2 \mathrm{t}$, onde $\mathrm{t}$ é o número de triângulos no grafo.

Definição 11 (Raio espectral). O raio espectral de um grafo $\mathrm{G}$, denotado por $\mu(\mathrm{G})$, é o número real não negativo $\mu(\mathrm{G}):=\operatorname{máximo}_{\{\mathrm{i}=1,2, \ldots, \mathrm{n}\}}\left\{\left|\lambda_{\mathrm{i}}\right|\right\}$, onde $\lambda_{1}, \lambda_{2}, \lambda_{3}, \ldots, \lambda_{\mathrm{n}}$ são os autovalores reais de G.

Portanto, o raio espectral de um grafo não orientado finito e conexo G é o raio do menor intervalo de centro na origem que contém todos os autovalores de $\mathrm{A}(\mathrm{G})$ (veja p. 38 em [1]). De fato, podemos ordenar os autovalores de $\mathrm{A}(\mathrm{G})$ (uma de suas matrizes de adjacência) da seguinte maneira $\lambda_{1} \geq \lambda_{2} \geq \lambda_{3} \geq \ldots \geq \lambda_{\mathrm{n}}$, visto que tais matrizes são simétricas e possuem todos os autovalores reais. Pelo Teorema de Perron-Fröbenius $\lambda_{1}>0$ e $\left|\lambda_{\mathrm{i}}\right| \leq \lambda_{1}$, assim o raio espectral é dado por $\mu(\mathrm{G})=\lambda_{1}$. Note que tal autovalor tem multiplicidades algébrica e geométrica iguais a 1, logo prova-se que $\mu(\mathrm{G})$ é o raio do menor intervalo de centro na origem que contém todos os autovalores de $\mathrm{A}(\mathrm{G})$ $[1]$.

Definição 12 (Clique). Um clique em um grafo é um subconjunto não vazio de seus vértices, tal que todos os vértices desse subconjunto são adjacentes entre si, ou seja, um clique é um subconjunto de vértices cujo subgrafo associado é completo. Define-se um k-clique como um clique com k vértices.

$\mathrm{Na}$ Figura 12 a seguir as arestas em vermelho que incidem sobre os vértices $\mathrm{v}_{3}, \mathrm{v}_{5}$ e $\mathrm{v}_{6}$ formam um 3-clique.

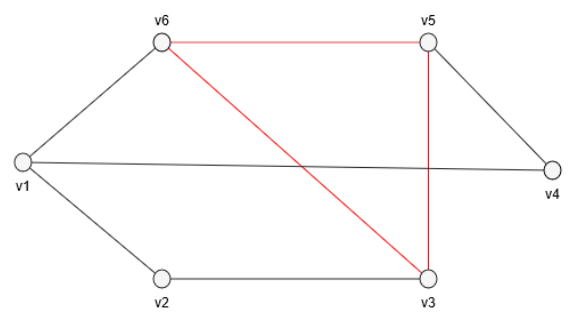

Figura 12: Clique.

Definição 13 (Clique máximo). Um clique com a maior cardinalidade possível em um grafo G é denominado de clique máximo do grafo $G$, representado por cl $(G)$.

O teorema a seguir proposto por [9] relaciona o clique máximo com o raio espectral de um grafo (Teorema 2).

Teorema 2 (Nikiforov). Seja G um grafo simples com $\lambda$ seu raio espectral e n seu número de vértices, então $\mathrm{cl}(\mathrm{G}) \geq \frac{\mathrm{n}}{\mathrm{n}-\lambda}$.

Definição 14 (Caminho de Hamilton). Seja G um grafo. Se existe um caminho que percorre todos os vértices de $\mathrm{G}$, ele é chamado de caminho de Hamilton. Um grafo que possui um caminho de Hamilton é chamado de grafo semi-hamiltoniano. 


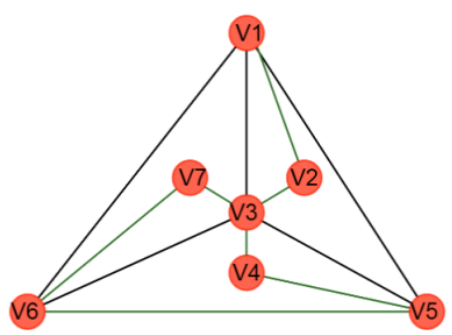

Figura 13: Grafo G e um caminho de Hamilton.

$\mathrm{Na}$ Figura 13 temos um caminho de Hamilton dado pela sequência $\mathrm{v}_{1} \mathrm{v}_{2} \mathrm{v}_{3} \mathrm{v}_{4} \mathrm{v}_{5} \mathrm{v}_{6} \mathrm{v}_{7}$.

Definição 15 (Grafo hamiltoniano). Seja G um grafo. Se, em G, existe um ciclo que contém todos os vértices de G, esse será chamado de ciclo de Hamilton. Um grafo é hamiltoniano se contém um ciclo de Hamilton.

As figuras 14, 15a e 15b ilustram exemplos de grafos hamiltonianos.

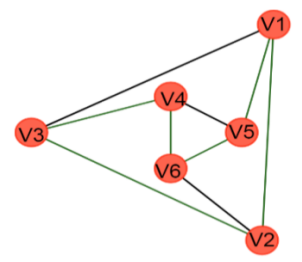

Figura 14: Grafo hamiltoniano G.

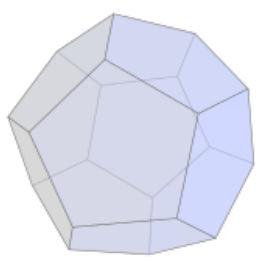

(a)

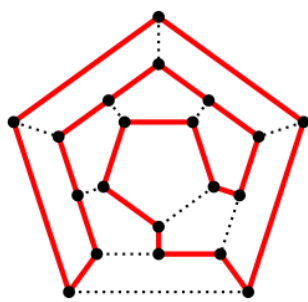

(b)

Figura 15: Dodecaedro regular e sua projeção no plano.

Fonte: https://pt.wikipedia.org/wiki/Ficheiro:Hamiltonian_path.svg.

Os grafos que estaremos interessados a partir de agora são todos não orientáveis, finitos, simples e conexos, já que grafos desconexos são claramente não hamiltonianos e não semi-hamiltonianos; além disso, se um grafo contém um subgrafo simples hamiltoniano, então o mesmo será hamiltoniano. 


\section{Três contraexemplos para a recíproca do Teorema de Fiedler-Nikiforov}

Nesta seção será exibida em cada contraexemplo uma realização geométrica dos grafos. Como dito anteriormente, os três objetos da definição precisa de um grafo não orientado são dados de forma implícita com as representações geométricas a seguir.

Contraexemplo 1. Seja D o grafo representado geometricamente abaixo, com ordenação de vértices dada por $\mathrm{v}_{1}<\mathrm{v}_{2}<\mathrm{v}_{3}<\mathrm{v}_{4}<\mathrm{v}_{5}<\mathrm{v}_{6}$, juntamente com sua matriz de adjacências $\mathrm{A}(\mathrm{D})$.

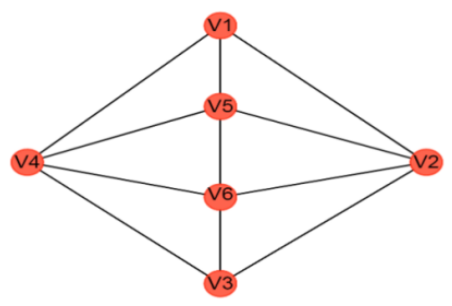

Figura 16: Grafo D.

$$
\mathrm{A}(\mathrm{D})=\left(\begin{array}{llllll}
0 & 1 & 0 & 1 & 1 & 0 \\
1 & 0 & 1 & 0 & 1 & 1 \\
0 & 1 & 0 & 1 & 0 & 1 \\
1 & 0 & 1 & 0 & 1 & 1 \\
1 & 1 & 0 & 1 & 0 & 1 \\
0 & 1 & 1 & 1 & 1 & 0
\end{array}\right)
$$

Como mostrado na Figura 16, o grafo D possui 6 vértices e não possui um k-clique para qualquer $\mathrm{k} \geq 4$. Portanto, $\mathrm{cl}(\mathrm{D})=3$. Aplicando o Teorema 2 de Nikiforov temos:

$3=\operatorname{cl}(\mathrm{D}) \geq \mathrm{n} /(\mathrm{n}-\lambda)=6 /(6-\lambda) \rightarrow 18-3 \lambda \geq 6 \rightarrow-3 \lambda \geq-12 \rightarrow \lambda \leq 4, \log o \mu(\mathrm{D})=\lambda \leq 4$.

Assim, utilizando-se o software Scilab para a matriz do grafo acima, temos os cálculos abaixo:

1. Polinômio característico:

$$
\begin{aligned}
\mathrm{p}_{\mathrm{D}}(\mathrm{x}) & =2.665 \times 10^{-15}+4 \mathrm{x}+5 \mathrm{x}^{2}-12 \mathrm{x}^{3}-11 \mathrm{x}^{4}+\mathrm{x}^{6} \\
& =4 \mathrm{x}+5 \mathrm{x}^{2}-12 \mathrm{x}^{3}-11 \mathrm{x}^{4}+\mathrm{x}^{6}
\end{aligned}
$$

2. Autovalores: $-2.2307254,-1.618034,-0.4828592,1.110 \times 10^{-16}, 0.618034$ e 3.7135847 .

Então o raio espectral do grafo $\mathrm{D}$ é dado por $\mu(\mathrm{D})=\lambda_{1}=3.7135847<\mathrm{n}-2=4$. Portanto nada se pode afirmar sobre a hamiltonicidade do grafo a partir do Teorema de Fiedler Nikiforov, mas o grafo contém um ciclo de Hamilton que, nesse caso, pode ser dado pelo ciclo $\mathrm{v}_{1} \mathrm{v}_{2} \mathrm{v}_{3} \mathrm{v}_{4} \mathrm{v}_{6} \mathrm{v}_{5} \mathrm{v}_{1}$. Logo, a recíproca de Fiedler-Nikiforov não é válida para o caso da desigualdade estrita.

Observação 1 . Note que o valor computacional encontrado no contraexemplo D é consistente com o resultado encontrado pelo Teorema 2 de Nikiforov já que $\mu(\mathrm{D})=\lambda \approx 3,7<4$. É interessante 
observar que o uso do clique máximo foi fundamental na construção de contraexemplos "pequenos" para a recíproca do Teorema 1, já que encontrar contraexemplos com 6 vértices (isto é, encontrar grafos hamiltonianos com 6 vértices cuja desigualdade fosse falsa) resumiu-se na busca por grafos de ordem 6 cujo clique máximo fosse igual a 3.

Contraexemplo 2. Seja E o grafo representado geometricamente abaixo, com ordenação de vértices dada por $\mathrm{v}_{1}<\mathrm{v}_{2}<\mathrm{v}_{3}<\mathrm{v}_{4}<\mathrm{v}_{5}<\mathrm{v}_{6}$, juntamente com sua matriz de adjacências $\mathrm{A}(\mathrm{E})$.

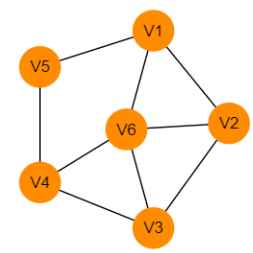

Figura 17: Grafo E.

$$
\mathrm{A}(\mathrm{E})=\left(\begin{array}{cccccc}
0 & 1 & 0 & 0 & 1 & 1 \\
1 & 0 & 1 & 0 & 0 & 1 \\
0 & 1 & 0 & 1 & 0 & 1 \\
0 & 0 & 1 & 0 & 1 & 1 \\
1 & 0 & 0 & 1 & 0 & 0 \\
1 & 1 & 1 & 1 & 0 & 0
\end{array}\right)
$$

Utilizando o software Scilab para a matriz do grafo E, temos os cálculos abaixo:

1. Polinômio característico:

$$
\begin{aligned}
p_{E}(x) & =-4+4 x+11 x^{2}-6 x^{3}-9 x^{4}-1.776 \times 10^{-15} x^{5}+x^{6} \\
& =-4+4 x+11 x^{2}-6 x^{3}-9 x^{4}+x^{6}
\end{aligned}
$$

2. Autovalores: $-2,-1.618034,-0.8608059,0.618034,0.7458983$ e 3.1149075 .

Então o raio espectral do grafo $\mathrm{E}$ é dado por $\mu(\mathrm{E})=\lambda_{1}=3.1149075<\mathrm{n}-2=4$. Note que o grafo E é hamiltoniano cujo ciclo é dado pela sequência $\mathrm{v}_{1} \mathrm{v}_{2} \mathrm{v}_{6} \mathrm{v}_{3} \mathrm{v}_{4} \mathrm{v}_{5} \mathrm{v}_{1}$. Encontramos outro contraexemplo, não isomorfo ao anterior (número de arestas diferente), para a recíproca do Teorema de Fiedler-Nikiforov.

Contraexemplo 3. Seja F o grafo representado geometricamente abaixo, com ordenação de vértices dada por $\mathrm{v}_{1}<\mathrm{v}_{2}<\mathrm{v}_{3}<\mathrm{v}_{4}<\mathrm{v}_{5}<\mathrm{v}_{6}$, juntamente com sua matriz de adjacências $\mathrm{A}(\mathrm{F})$. $A$ diferença entre o grafo $\mathrm{E} e \mathrm{~F}$ é que o grafo $\mathrm{F}$ contém uma aresta a mais que $\mathrm{E}$, a aresta $\mathrm{v}_{5} \mathrm{v}_{6}$. 


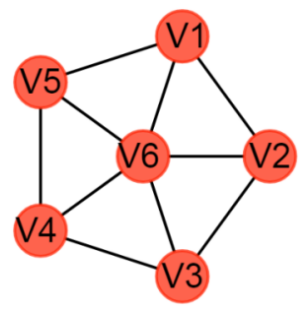

Figura 18: Grafo F.

$$
\mathrm{A}(\mathrm{F})=\left(\begin{array}{cccccc}
0 & 1 & 0 & 0 & 1 & 1 \\
1 & 0 & 1 & 0 & 0 & 1 \\
0 & 1 & 0 & 1 & 0 & 1 \\
0 & 0 & 1 & 0 & 1 & 1 \\
1 & 0 & 0 & 1 & 0 & 1 \\
1 & 1 & 1 & 1 & 0 & 0
\end{array}\right)
$$

Utilizando o software Scilab para a matriz do grafo F, temos os cálculos abaixo:

1. Polinômio característico:

$$
\begin{aligned}
\mathrm{p}_{\mathrm{F}}(\mathrm{x}) & =-5+8 \mathrm{x}+10 \mathrm{x}^{2}-10 \mathrm{x}^{3}-10 \mathrm{x}^{4}+1.7 \times 10^{-15} \mathrm{x}^{5}+\mathrm{x}^{6} \\
& =-5+8 \mathrm{x}+10 \mathrm{x}^{2}-10 \mathrm{x}^{3}-10 \mathrm{x}^{4}+\mathrm{x}^{6}
\end{aligned}
$$

2. Autovalores: $-1.618034,-1.618034,-1.4494897,0.618034,0.618034$ e 3.4494897.

Então o raio espectral do grafo $\mathrm{F}$ é dado por $\mu(\mathrm{F})=\lambda_{1}=3.4494897<\mathrm{n}-2=4$. Note que o grafo $\mathrm{F}$ é hamiltoniano cujo ciclo é dado pela sequência $\mathrm{v}_{1} \mathrm{v}_{2} \mathrm{v}_{3} \mathrm{v}_{4} \mathrm{v}_{5} \mathrm{v}_{6} \mathrm{v}_{1}$. Encontramos outro contraexemplo, não isomorfo aos anteriores (número de arestas diferente), para a recíproca do Teorema de Fiedler-Nikiforov.

\section{O Teorema de Bolzano e a não validade da recíproca de Fiedler-Nikiforov}

Nesta seção será verificado que os cálculos obtidos a partir da ferramenta computacional Scilab são consistentes. Para tanto usou-se o Teorema de Bolzano e o software gráfico GeoGebra para a verificação dos resultados aqui apresentados. Nesta seção denote por (c; d) um intervalo aberto da reta com extremos $\mathrm{c}<\mathrm{d}$.

Teorema 3 (Existência de raízes). Seja $\mathrm{f}:[\mathrm{a} ; \mathrm{b}] \rightarrow \mathbb{R}$ uma função de variável real $\mathrm{x}$ e contínua. Suponha que $\mathrm{f}(\mathrm{a}) . \mathrm{f}(\mathrm{b})<0$, então existe um $\mathrm{z} \in(\mathrm{a} ; \mathrm{b})$ tal que $\mathrm{f}(\mathrm{z})=0$.

Como é conhecido, cada polinômio é uma função contínua na reta; logo, para aplicarmos o Teorema de Bolzano para essas funções basta verificar as mudanças de sinais da função. Vamos estudar agora o comportamento geométrico do polinômio característico do grafo D da Figura 16. O gráfico desse polinômio encontra-se na Figura 19. 


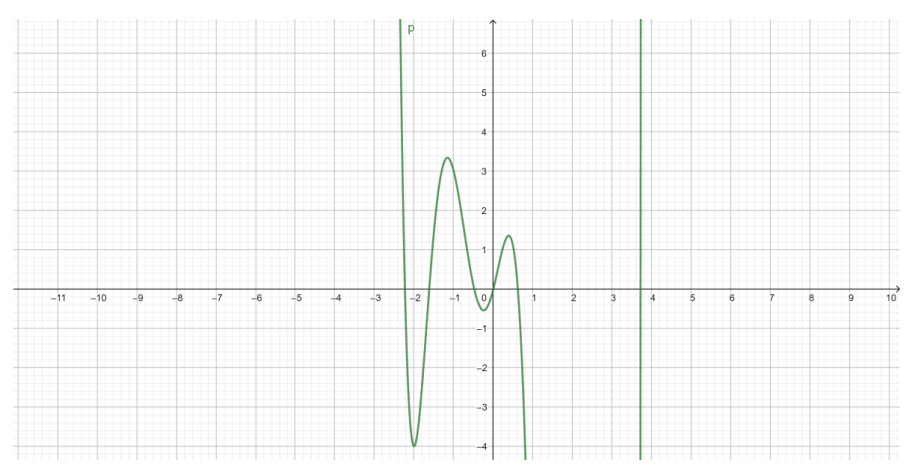

Figura 19: Gráfico do polinômio de A(D).

Note que $\mathrm{p}_{\mathrm{D}}(-3)=195 ; \mathrm{p}_{\mathrm{D}}(-2)=-4 ; \mathrm{p}_{\mathrm{D}}(-1)=3 ; \mathrm{p}_{\mathrm{D}}(-0,1)=-0,339099 ; \mathrm{p}_{\mathrm{D}}(0)=0 ; \mathrm{p}_{\mathrm{D}}(0,1)=$ 0,$436901 ; \mathrm{p}_{\mathrm{D}}(1)=-13 ; \mathrm{p}_{\mathrm{D}}(3)=-429 ; \mathrm{p}_{\mathrm{D}}(4)=608$. Observe que 0 é uma raiz desse polinômio. Aplicando o Teorema de Bolzano confirmamos que os autovalores distintos e não nulos do polinômio característico encontram-se nos seguintes intervalos abertos respectivamente: $(-3 ;-2),(-2 ;-1)$, $(-1 ;-0,1),(0,1 ; 1)$ e $(3 ; 4)$. Donde concluímos que o raio espectral determinado no contraexemplo 1 é coerente com o resultado encontrado pelo Teorema de Bolzano visto que $\mu(\mathrm{D})=\lambda_{1}=3.7135847 \in$ $(3,4)$ o que implica que $\mu(\mathrm{D})<4$.

Agora vamos estudar o comportamento geométrico e algébrico do polinômio característico da matriz de adjacência escolhida para o grafo E do contraexemplo 2 da seção anterior. O gráfico deste polinômio encontra-se na Figura 20.

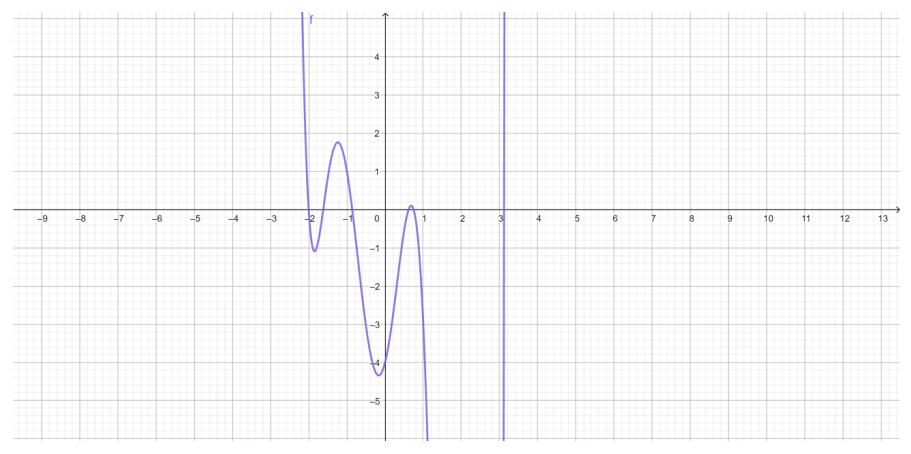

Figura 20: Gráfico do polinômio de A(E).

Note que $\mathrm{p}_{\mathrm{E}}(-2)=0 ; \mathrm{p}_{\mathrm{E}}(-1,9)=-0,979019 ; \mathrm{p}_{\mathrm{E}}(-1)=1 ; \mathrm{p}_{\mathrm{E}}(0)=-4 ; \mathrm{p}_{\mathrm{E}}(1)=-3 ; \mathrm{p}_{\mathrm{E}}(0,7)=$ 0,$088749 ; \mathrm{p}_{\mathrm{E}}(1)=-3 ; \mathrm{p}_{\mathrm{E}}(3)=-55 ; \mathrm{p}_{\mathrm{E}}(4)=1596$. Observe que -2 é uma raiz desse polinômio. Aplicando o Teorema de Bolzano confirmamos que os 5 outros autovalores distintos do polinômio característico encontram-se nos seguintes intervalos abertos respectivamente: $(-1,9 ;-1),(-1 ; 0)$, $(0 ; 0,7),(0,7 ; 1)$ e $(3 ; 4)$. Donde concluímos que o raio espectral determinado no contraexemplo 2 é coerente com o resultado encontrado pelo Teorema de Bolzano, visto que $\mu(\mathrm{E})=\lambda_{1}=3.1149075 \in$ $(3,4)$ o que implica que $\mu(\mathrm{E})<4$. 
Por fim, vamos estudar o comportamento geométrico do polinômio característico da matriz de adjacência escolhida para o grafo $\mathrm{F}$ do contraexemplo 3 da seção anterior. Os gráficos desse polinômio se encontram nas figuras 21 e 22.

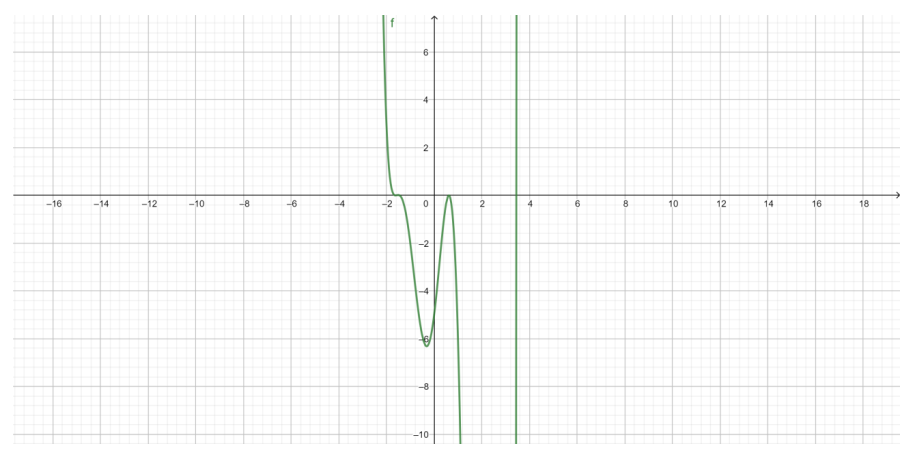

Figura 21: Gráfico do polinômio de $\mathrm{A}(\mathrm{F})$.

Seguem também algumas ampliações do gráfico anterior.

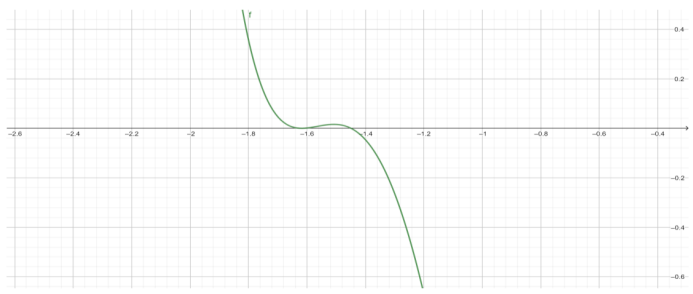

Figura 22: Gráfico Ampliado.

Para discutirmos o caso do polinômio da matriz $\mathrm{F}$ vamos usar o seguinte resultado devido a Lagrange e que pode ser encontrado na página 39, Proposição 3 do livro de Young [17] (a demonstração é feita usando-se $\left.\mathrm{a}_{\mathrm{n}}=1, \mathrm{M}=-\mathrm{P}\right)$.

Proposição 2 (Teorema de Lagrange. Limite superior para raízes positivas). Seja $\mathrm{p}(\mathrm{x})=\mathrm{a}_{\mathrm{n}} \mathrm{x}^{\mathrm{n}}+$ $\mathrm{a}_{\mathrm{n}-1} \mathrm{x}^{\mathrm{n}-1}+\mathrm{a}_{\mathrm{n}-2} \mathrm{x}^{\mathrm{n}-2}+\ldots+\mathrm{a}_{1} \mathrm{x}+\mathrm{a}_{0}=0$, uma equação polinomial de grau $\mathrm{n}$. Suponha que $\mathrm{a}_{\mathrm{n}}>0 e$ $\mathrm{a}_{0} \neq 0$. Então um limite superior para as raízes positivas da equação dada, caso existam, é dado pelo número: $\mathrm{L}=1+\sqrt[\mathrm{n}-\mathrm{k}]{\frac{\mathrm{M}}{\mathrm{a}_{\mathrm{n}}}}$, onde $\mathrm{k}$ é o grau do maior monômio com coeficiente negativo e $\mathrm{M}$ é o módulo do menor coeficiente negativo no polinômio

Para matrizes de grafos não orientáveis finitos e conexos, a garantia de raízes positivas é dada pelo Teorema de Perron-Fröbenius. Assim o polinômio característico de F terá raízes positivas e o raio espectral deverá ser menor que o valor de L pela Proposição 2. Para o polinômio $\mathrm{p}_{\mathrm{F}}(\mathrm{x})=$ $-5+8 x+10 x^{2}-10 x^{3}-10 x^{4}+x^{6}$ obtemos para $L$ o seguinte valor $L=1+\sqrt[6-4]{\frac{10}{1}}=1+\sqrt{10} \approx 4,16$ 
Teoricamente sabemos que $\mu(\mathrm{F})<\mathrm{L} \approx 4,16$. No entanto, analisando o gráfico da Figura 21 notamos que $\mathrm{p}_{\mathrm{D}}(3)=-242<0 ; \mathrm{p}_{\mathrm{D}}(4,16)>\mathrm{p}_{\mathrm{D}}(4)=1083>0$ e o polinômio é crescente em $(3, \infty)$. Portanto, existe uma única raiz de $\mathrm{p}_{\mathrm{D}}(\mathrm{x})$, digamos $\xi$, tal que $\xi \in(3 ; 4,16), \operatorname{logo} \xi=\mu(\mathrm{F})$. Pelo Teorema de Bolzano existe uma raiz no intervalo $(3 ; 4) \subset(3 ; 4,16)$ o que mostra que $\xi=\mu(\mathrm{F})<4$. Assim, o resultado computacional encontrado no contraexemplo 3 da seção anterior é coerente com a resposta analítica.

A estratégia para justificar a veracidade dos valores encontrados pelo computador foi diferente no terceiro contraexemplo, nos dois primeiros usamos uma metodologia conhecida como isolamento de raízes; essa, por sua vez, utiliza o Teorema de Bolzano. Em seguida, observou-se que todas as raízes eram distintas nos dois primeiros, contudo no terceiro caso o polinômio apresentou dois pares de raízes iguais, porém os pares eram distintos um dos outro. Logo, a aplicação direta do Teorema de Bolzano só permitiu localizar duas das seis raízes do terceiro polinômio, e não foi possível saber onde se localizava o raio espectral de forma imediata. Contudo, após a aplicação do Teorema de Lagrange, pode-se usar o Teorema de Bolzano para isolar a raiz de maior valor positivo, isto é, o raio espectral do grafo.

\section{Considerações finais}

Nas duas primeiras seções deste trabalho apresentamos conceitos, exemplos e resultados básicos sobre a teoria de grafos que foram utilizados no entendimento e desenvolvimento da questão central deste artigo, que foi a construção dos contraexemplos para a recíproca do Teorema 1 de FiedlerNikiforov. De forma mais clara, o foco principal foi a obtenção de grafos hamiltonianos cujo raio espectral fosse menor do que o número de vértices do grafo menos duas unidades. Na seção 3, esses contraexemplos são apresentados. Usando-se o resultado do Teorema 2 de Nikiforov (sobre clique máximo em grafos), escolheram-se alguns grafos com 6 vértices, tendo clique máximo igual a 3 para serem investigados. Alguns foram descartados e três deles foram selecionados. Posteriormente, na seção 4, constatou-se com o uso do Scilab que esses eram contraexemplos para o resultado supracitado. No Scilab, foram usados alguns comandos para determinar os polinômios característicos e os autovalores da matriz de adjacência dos grafos; em especial, os mais utilizados foram o "poly" e o "spec".

Vale ressaltar que, devido à natureza numérica das ferramentas utilizadas, alguns dos valores obtidos representam aproximações, e em alguns casos foi necessário usar certos conhecimentos teóricos para fazer ajustes tanto nos polinômios característicos quanto no cálculo dos autovalores. Esse detalhe é interessante pois também torna evidente a importância de uma ferramenta computacional para sugerir respostas que algumas vezes devem ser melhoradas com o uso da teoria. Através dos polinômios característicos encontrados pelo Scilab foi possível localizar os autovalores aplicandose o Teorema de Bolzano- o que proporcionou uma verificação teórica de que os contraexemplos encontrados na seção 3 eram satisfatórios, embora a ferramenta computacional já tivesse mostrado a validade dos mesmos. O uso dos gráficos dos polinômios característicos fornecidos pelo GeoGebra foi essencial neste estudo. Isso foi particularmente evidenciado no contraexemplo 2, no qual, através das informações gráficas, pode-se escolher os números $-1,9$ e -1 , de tal maneira que $\mathrm{P}_{\mathrm{E}}(-1,9)<0$ e $\mathrm{P}_{\mathrm{E}}(-1)>0$, o que implicou a existência de uma raiz no intervalo $(-1,9 ;-1)$. No contraexemplo 3 não foi possível localizar todas as raízes diretamente pelo Teorema de Bolzano, mas, utilizando-se das informações gráficas e do Teorema de Lagrange, constatou-se que o raio espectral pertencia ao intervalo $(3,4)$.

Ficou evidente no desenvolvimento deste estudo que o uso de ferramentas computacionais pode e 
deve ser utilizado na elaboração, teste e validação de resultados matemáticos como os que foram apresentados neste texto. Além disso, tais passos ilustram como a teoria matemática e o uso de recursos computacionais podem se relacionar de maneira harmoniosa.

Após este estudo, surgiram algumas questões que não foram resolvidas até o momento:

Questão 1. Uma análise comparativa entre os grafos E e F mostrou que ao retirar uma das arestas de F, foi encontrado um novo grafo, chamado de E, que possui uma matriz de adjacência que contém autovalores não repetidos, porém mantiveram-se os autovalores que eram múltiplos para a matriz de adjacência do grafo F: os autovalores -1,618034 e 0,618034. Essa "perturbação" sempre provoca a quebra da multiplicidade dos autovalores? Para que tipo de aresta devemos fazer isso? Ou foi uma coincidência gerada por esses exemplos?

Questão 2. Existem relações entre as raízes múltiplas e os tipos de grafos? O que acontece com raízes que não são múltiplas?

Questão 3. Existe uma condição para que o 0 seja um autovalor de uma matriz de adjacência de um determinado grafo? Quais propriedades têm que ter um grafo, para que esse possua um autovalor igual a 0 ?

\section{Agradecimentos}

Agradecemos ao apoio dado pelo ICT-UFVJM, além do auxílio dado pelo Programa Picme-UFMG, que em parceria com a UFVJM possibilitou o desenvolvimento deste trabalho disponibilizando uma bolsa de estudos à aluna colaboradora desse artigo. Por fim, agradecemos as correções sugeridas pelo parecerista e a atenção que nos foi dada pelo editor.

\section{Referências}

[1] Abreu, N.; Del-Vecchio, R.; Trevisan, V.; Vinagre, C. Teoria espectral de grafos - Uma introdução. III Colóquio de Matemática da Região Sul (Florianópolis), SC, 2014.

[2] Bondy, J. A.; Murty, U. S. R. Graph Theory with Applications. North Holland, 5th ed. New York, 1982.

[3] Cvetković, D.; Rowlinson, P.; Simić, S. Eigenspaces of graphs. Encyclopedia of Mathematics and its applicatons. Cambridge University Press (66), 1997.

[4] Fiedler, M.; Nikiforov, V. Linear Algebra and its Applications. Elsevier Science Publishing Co. Inc., v. 432, p. 2170-2173, 2010.

[5] Geogebra. GeoGebra - Powerfull, Free Online Graphing Calculator and Interactive Geometry. 2017. Disponível em: <http://www.geogebra.org/>. Acesso em: fevereiro de 2020.

[6] Graphviz. Version 2.43.0. Graphviz - Graph Visualization Software. Disponível em: <https: //graphviz.org/>. Acesso em: fevereiro de 2020.

[7] Harary, F. Graph Theory. Reading, Massachusetts - Menlo Park: Addison Wesley Publishing Co. Inc., 1969.

[8] Kolman, B.; Hill, D. R. Introdução à Álgebra Linear com Aplicações. LTC-GEN, 8. ed. (Rio de Janeiro), 2006.

[9] Nikiforov, V. Some inequalities for the largest eigenvalue of a graph. Combinatorics, Probability and Computing, v. 11, p. 179-189, 2002. 
[10] Nicoletti, M. C.; Hruschka Júnior, E. R. Fundamentos da Teoria dos Grafos para Computação. EDUFSCar (São Carlos), 2006.

[11] Santos, M. de S. Ciclos Hamiltonianos em Grafos. Ciência e Natura, Santa Maria, v. 39, p. 595-626, 2017.

[12] Scilab. Version 6.0.1. ESI Group, 2017. Disponível em: <https://www.scilab.org/download/ 6.0.1 >. Acesso em: abril de 2020.

[13] Wikipedia. Dodecaedro. Disponível em: <https://es.wikipedia.org/wiki/Usuario: Dodecaedro>. Acesso em: abril de 2020.

[14] Wikipedia. Grafo das ilhas de Königsberg. Disponível em: <https://pt.wikipedia.org/wiki/ Ficheiro:K\%C3\%B6nigsberg_graph.png>. Acesso em: abril de 2020.

[15] Wikipedia. Königsberg Bridges. Disponível em: <https://en.wikipedia.org/wiki/File: Konigsberg_bridges.png>. Acesso em: abril de 2020.

[16] Yed Graph Editor. Version 3.18.2. Works the diagramming company, 2018. Disponível em: <https://www.yworks.com/products/yed/download\#download>. Acesso em: abril de 2020.

[17] Young, J. R. Theory and Solution of algebraical equations with the recente researches of Budan, Fourier and Sturm, on the separation of the real and imaginary roots of equations. John Souter, 73, ST Paul's Church-Yard (London), 1835.

[18] Xavier, M. F. R. Um Teorema de Fiedler-Nikiforov sobre a Hamiltonicidade de Grafos Simples e Conexos. 61 p. Dissertação (Trabalho de Conclusão de Curso) — Universidade Federal dos Vales do Jequitinhonha e Mucuri, Diamantina-MG, 2019.

Anderson Luiz P. Porto Universidade Federal dos Vales do Jequitinhonha e Mucuri <ander.porto@ict.ufvjm.edu.br>

Marcelle Fernanda R. Xavier Universidade Federal de Minas Gerais <marcellefernanda1@gmail.com>

Douglas F. G. Santiago Universidade Federal dos Vales do Jequitinhonha e Mucuri <douglas.santiago@ict.ufvjm.edu.br>

Recebido: 21/04/2020

Publicado: 15/03/2021 